\title{
Acneiform lesions secondary to ZD1839, an inhibitor of the epidermal growth factor receptor
}

\author{
M. Fernandez-Galar, A. España and J. M. López-Picazo* \\ Departments of Dermatology and *Oncology, University Clinic of Navarra, Pamplona, \\ Spain
}

Correspondence: A. España, Department of Dermatology, University Clinic of Navarra, PO Box 4209, 31080 Pamplona, Spain.

\section{SUMMARY}

Drugs that inhibit the epidermal growth factor receptor, such as ZD1839 or C225, are being used increasingly in the treatment of solid tumours. This has led to the appearance of new secondary effects. We describe the case of a patient who presented with an acneiform eruption secondary to the administration of ZD1839. These lesions healed in a few days after stopping the drug.

\section{REPORT}

Novel chemotherapeutic agents may produce cutaneous side effects. Monoclonal antibodies to the epidermal growth factor receptor (EGF-R) and EGF-R-specific receptor tyrosine kinase inhibitors are in use in clinical trials for the treatment of solid tumours. Among available anti-EGF-R antibodies the most advanced in clinical development is C225 (Cetuximab). Furthermore there is a large number of inhibitors of the EGF-R tyrosine kinase under clinical development. Gefitinib, also known as ZD1839 (Iressa ${ }^{\mathrm{TM}}$, Astra-Zeneca, Wilmington, DE, USA) is a low-molecular weight quinazolin derivative that inhibits the activation of EGF-R tyrosine kinase through competitive binding of the ATP-binding domain of the receptor. It has recently been approved by the Food and Drug Administration as monotherapy for the treatment of patients with locally advanced or metastatic nonsmall-cell lung cancers (NSCLCs) after failure of both platinum-based and docetaxel chemotherapies. ${ }^{1}$

We present the case of a 50-year-old male diagnosed with lung adenocarcinoma in 1997. He received surgical treatment (lobectomy) and chemotherapy (paclitaxel, cisplatin, gemcitabine, irinotecan, vinorelbine, docetaxel, oxaliplatin, at different periods), and adjuvant radiotherapy for successive disease recurrences. In December 2001, the patient started treatment with ZD1839 at $200 \mathrm{mg}$ daily. Seven days after starting this treatment, the patient presented with pustules and erythematous papules on the face, trunk, back and presternal area. The patient continued with the treatment and after 2 months, because his lesions still persisted, he came to our department. Physical examination revealed a papulopustular eruption, located on the seborrhoeic areas of the face (Fig. 1a) and on the anterior and posterior aspects of the trunk (Fig. 1b), with no comedones or cysts. Bacteriology and mycology cultures of these lesions were negative. During this period the patient was not receiving any other drugs. A biopsy from an active lesion revealed orthokeratotic hyperkeratosis of the stratum corneum, lymphocyte 
exocytosis in the basal layer of the epidermis, with basal layer hydropic degeneration. A dense inflammatory infiltrate of lymphocytes and neutrophils was observed in the dermal papillae. Furthermore, there was a perifollicular dense aggregate of multinucleated giant cells and macrophages (Fig. 2). Direct immunofluorescence was negative for immunoglobulins, fibrinogen and complement. The patient was diagnosed as having an acneiform eruption secondary to ZD1839 administration. The drug was stopped, and the skin lesions resolved over a 2-week period.

Blocking the EGF-R inhibits keratinocyte proliferation and migration ${ }^{2}$ and induces apoptosis. ${ }^{3}$ The EGF-R has also been implicated in keratinocyte differentiation. ${ }^{4}$ Drugs that inhibit the EGF-R could also possibly be useful in the treatment of other diseases where EFG-R is implicated, such as psoriasis, ${ }^{5}$ or epithelial skin tumours. ${ }^{6}$

In some histologic studies of patients treated with ZD1839 or C225, keratin plugs and microorganisms have been found in dilated infundibula. ${ }^{7-10}$ These changes are probably secondary to an aberrant differentiation of suprabasal keratinocytes, caused by EGF-R inhibition, which results in an acneiform eruption. Infundibular necrosis together with alopecia were observed in EGF-R knockout mice, suggesting a central role for EGF-R also in hair follicle biology. ${ }^{11}$

EGF-R inhibitors are being increasingly used in the treatment of solid tumours. Side effects described include acneiform lesions as in our patient, ${ }^{7,8}$ seborrhoeic dermatitis, paronychia $^{12}$ necrolytic migratory erythema-like lesions ${ }^{13}$ acute interstitial pneumonia ${ }^{14}$ and gastrointestinal symptoms. Busam et al. ${ }^{7}$ studied a series of 10 patients with metastatic renal carcinoma treated with C225 for 1 week: all 10 patients developed a follicular rash in an acneiform distribution. This was accompanied by pain and fisuring of the distal finger tips. No infectious cause was found in these patients. Two patients had oral ulcers of 2-3 mm, with negative cultures for herpes virus. Our patient did not have oral or finger tip lesions. Furthermore, Van Doorn et al. ${ }^{8}$ recently described an acneiform eruption in three patients receiving treatment with ZD1839. These patients presented with follicular papules and pustules in an acneiform distribution together with generalized cutaneous xerosis and scaling and altered hair growth. Bacteriologic and mycologic cultures were performed in all three patients, and Propionibacterium acnes was found in one patient. In summary, we describe a further case of a florid acneiform eruption secondary to ZD1839. Many of the novel anti-inflammatory and chemotherapeutic agents target the EGF-R or related signalling pathways. Dermatologists need to be aware of these agents and their cutaneous side effects.

\section{REFERENCES}

1. John Mendelsohn Jose Baselga. Status of epidermal growth factor receptor antagonists in the biology and treatment of cancer. J Clin Oncol 2003; 21: 278799.

2. Baselga J, Averbuch SD. ZD1839 (Iressa) as an anticancer agent. Drugs 2000; 60 (Suppl. 1): 33-40.

3. Csaba Kari Tung O, Rocha de Quadros, CM et al. Targeting the epidermal growth factor receptor in cancer: apoptosis takes center stage. Cancer Res 2003; 63: $1-5$.

4. Jost M, Kari C, Rodeck U. The EGF receptor an essential regulator of multiple epidermal functions. Eur J Dermatol 2000; 10: 505-10. 
5. Ben-Bassat H. Biological activity of tyrosine kinase inhibitors: novel agents for psoriasis therapy. Curr Opin Invest Drugs 2001; 2: 1539-45.

6. Hansen LA, Woodson IIRL, Holbus $\mathrm{S}$ et al. The epidermal growth factor receptor is required to maintain the proliferative population in the basal compartment of epidermal tumors. Cancer Res 2000; 60: 3328-32.

7. Busam KJ, Capodieci P, Motzer R et al. Cutaneous sideeffects in cancer patients treated with the antiepidermal growth factor receptor antibody C225. Br J Dermatol 2001; 144: 1169-76.

8. Doorn RV, Kirtschig G, Scheffer E et al. Follicular and epidermal alterations in patients treated with ZD1839 (Iressa), an inhibitor of the epidermal growth factor receptor. Br J Dermatol 2002; 147: 598-601.

9. Kimsay-Asadi A, Jih MH. Follicular toxic effects of chimeric anti-epidermal growth factor receptor antibody Cetuximab used to treat human solid tumors. Arch Dermatol 2002; 138: 129-31.

10. Albanell J, Rojo F, Averbuch S et al. Pharmacodynamic studies of the epidermal growth factor receptor inhibitor ZD1839 in skin from cancer patients: histopathologic and molecular consequences of receptor inhibition. J Clin Oncol 2002; 20: 110-24.

11. Hansen LA, Alexander N, Hogan ME et al. Genetically null mice reveal a central role for epidermal growth factor receptor in the differentiation of the hair follicle in normal hair development. Am J Pathol 1997; 150: 1959-75.

12. Nakano J, Nakamura M. Paronychia induced by Gefitinib, an Epidermal growth factor receptor tyrosine kinase inhibitor. J Dermatol 2003; 30: 261-2.

13. Trojan A, Jacky E, Follath F, Dummmer R. Necrolytic migratory erythema (Glucagonoma)-like skin lesions induced by EGF-receptor inhibition. Swiss Med Wkly 2003; 133: 22.

14. Inoue A, Saijo Y, Maemondo M, Gomi K. Severe acute interstitial pneumonia and gefitinib. Lancet 2003; 361: 137-9. 

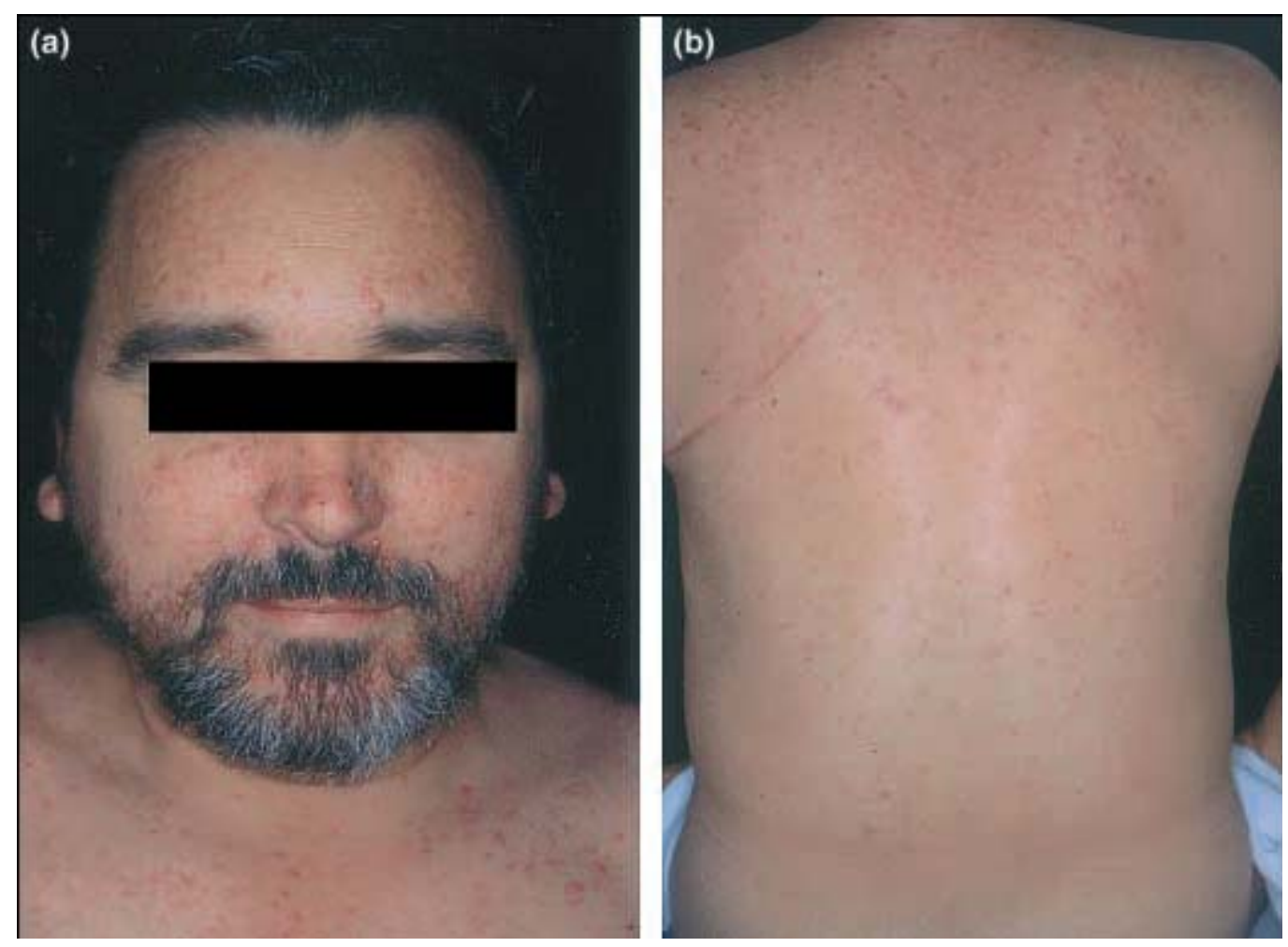

Figure 1. (a) Papular-pustular lesions on the seborrhoeic areas of the face; (b) Papularpustular lesions also present on the patient's back. 


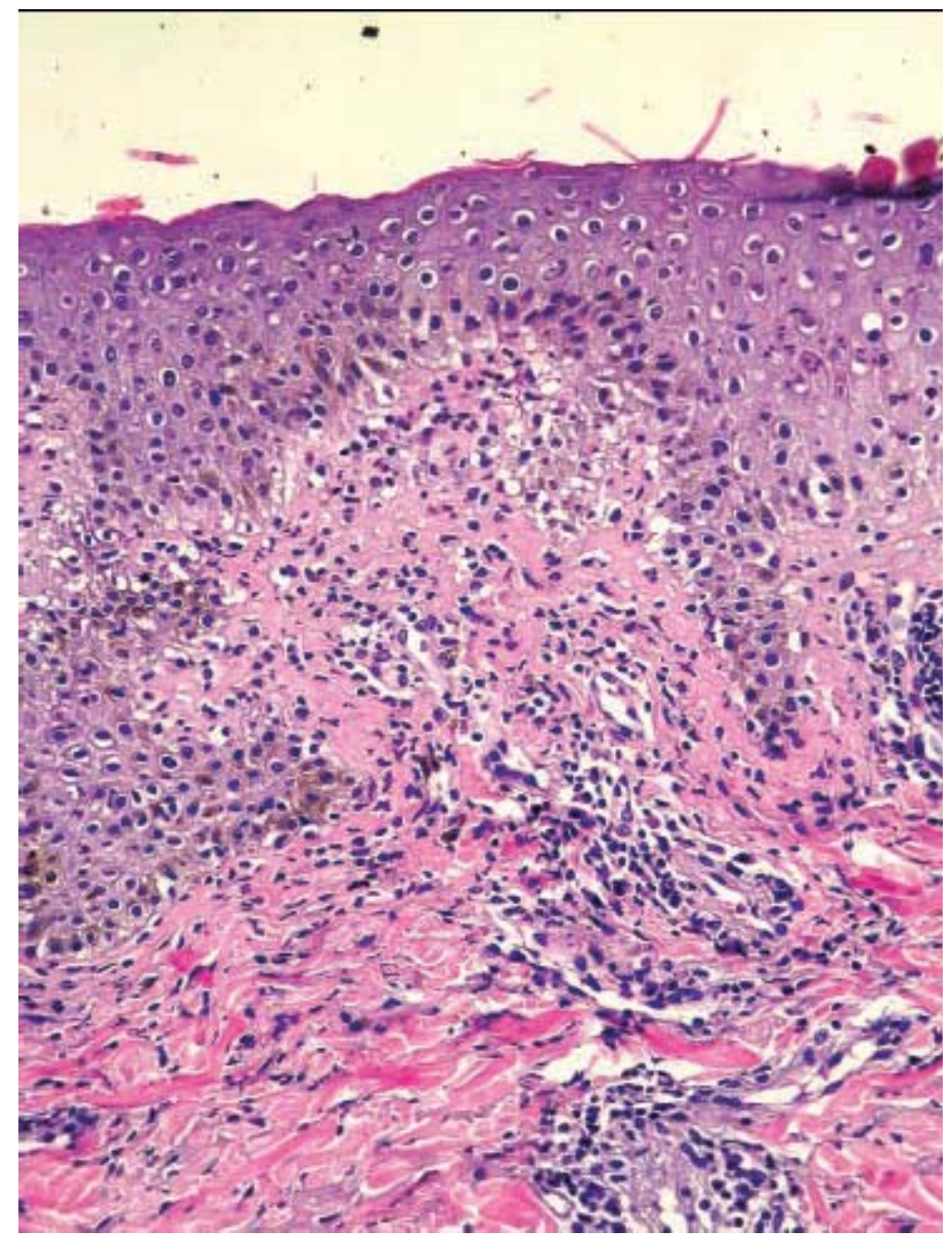

Figure 2. Orthokeratotic hyperkeratosis and lymphocytic exocytosis with hydropic degeneration of the basal layer. There is a dense inflammatory infiltrate in the dermal papillae and a perifollicular aggregate of multinucleated giant cells and macrophages. 